\title{
Numerical Analysis of Polymeric Thin Films Fracture Based on Energetic Approach "The Essential Work of Fracture"
}

\author{
Mohamed Belhamiani ${ }^{1 *}$, Mohamed Benguediab², Wahid Oudad ${ }^{1}$ \\ 1 Smart Structures Laboratory (SSL), Department of Mechanical Engineering, Belhadj Bouchaib University Center, \\ P. O. B. 284,46000 Ain Témouchent, Algeria \\ 2 Department of Mechanical Engineering, Faculty of Technology, Université Djillali Liabes - Sidi Bel Abbès, \\ P. O. B. 89, 22000 Sidi Bel Abbès, Algeria \\ *Corresponding author, e-mail: mbelhamiani@gmail.com
}

Received: 25 June 2018, Accepted: 15 October 2019, Published online: 04 November 2019

\begin{abstract}
Actually, polymeric materials are widely used in industry due to their attractive properties. These materials are replacing the traditional materials used to manufacture mechanical components. Thus, their mechanical behavior should be known for a better and judicious use of the material. Fracture mechanics principles and tools are used to characterize polymeric thin films fracture. In this paper a global energetic approach proposed to characterize the rupture phenomenon and determine the essential work of fracture.
\end{abstract}

\section{Keywords}

polymeric materials, films, rupture, essential work of fracture, finite element method

\section{Introduction}

Among the modes of damage of structures, rupture is the phenomenon the most dangerous due to its suddenless which makes difficult to plan or take preventive action. That is why, the knowledge and modeling of rupture have a bigger interest among industrials whishing better computational means for prediction.

Polymeric material rupture is a complex problem. In many cases, one has guaranteed their resistance by over dimensioning which gives a sufficient safety margin. This type of approach cannot be applied in industries such as energy transportation. High reliability structure, which require higher safety. Instead of introducing an empirical safety coefficient at all levels (design, manufacturing and operations) it is useful that an efficient method should be developed to perform safety computations and predict the behavior of structure in the most unfavorable case. The safety margin can be then easily known.

In this paper, a numerical analysis of polymeric thin films rupture is carried out on SENT specimen using the essential work of fracture. The mechanical conditions of validations in terms of ligament lengths have also been studied.

The results obtained prove that $w_{e}$ and $\beta w_{p l}$, can be evaluated using two distinct intrinsic ratios similar to than presented by Cotterel and Reddel [1] but another method has been established to determine that.

\section{Theoretical principle}

Broberg proposes [2], in the case of a material presenting plasticity, to separate the non-elastic region at the crack tip into two distinct regions as shown in Fig. 1:

- The first zone called "internal zone" or "end zone" in which the process of rupture initiates. This zone

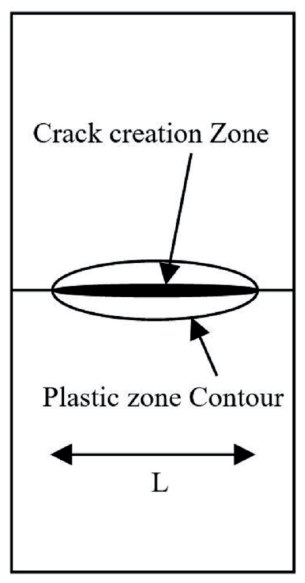

Fig. 1 Schematic representation of zone separation size. 
is in fact the cracks zone referred to as fracture process zone (F.P.Z).

- The second zone: external "outer zone" which entour the crack elaboration zone referred to as peripheral zone.

From Broberg's idea, many researchers [3-7] had characterized ductile rupture of materials by partitioning the total work of fracture $W_{f}$ by:

- The work dissipated in the F.P.Z, $W_{e}$ which considered essential in the rupture process.

- The work that is responsible for plastic deformation $W_{p l}$ which is considered secondary in rupture process. This work is dissipated in order to deform the material in the external in the external plastic zone of the F.P.Z.

$W_{f}=W_{e}+W_{p l}$

$W_{e}:$ is the total essential work. It corresponds to the integral of the essential work during the evolution of the work, it can be considered proportional to the size of the ligament $L$ and to the area of cracked ligament. $W_{p l}$ : is the specific non-essential work of rupture i.e. the work dissipated per unit volume of material.

By taking Eq. (1) and dividing the area of ligament, we obtain:

$w_{f}=w_{e}+\beta L w_{p l}$

$w_{f}$ : is the total specific work of rupture, i.e. the total work of fracture per unit area of ligament. The term $\beta w_{p l}$ is not considered as a material property but as a measure of work of plastic deformation around the crack tip Eq. (2) gives a linear relation between $w_{f}$ and $L$ (Fig. 2). The slope of this relation is proportional to $w_{p l}$. For a given material, $w_{p l}$ increases with ductility.

For relation Fig. 2 to be applied, the ligament must be in plane stress state. Many writers in literature [1]

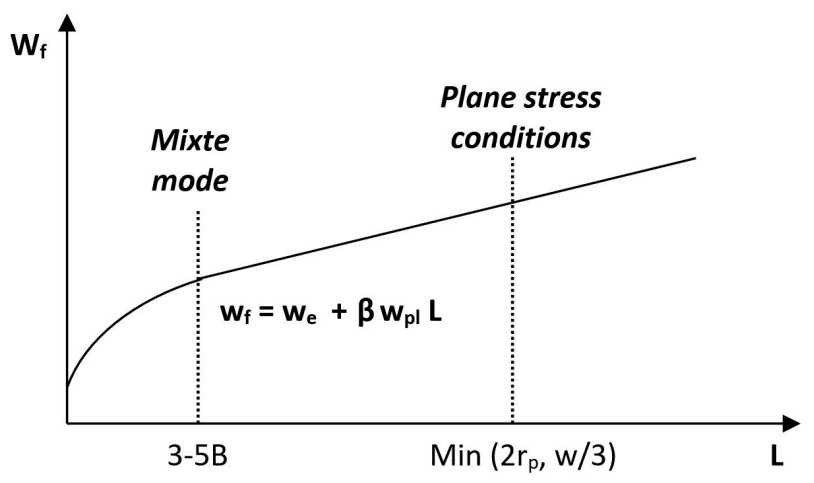

Fig. 2 Linear relation between total specific work and initial ligament length. and [5-8] suggest maintaining the ligament length 3 to 5 times the thickness of the specimen such that:

$L^{*}=3-5 B<L$.

That plastic zone at the crack tip must contain the entire ligament, such that it will be completely plasticized before the crack propagates. This condition must be fulfilled in order to maintain the proportionality of $w_{p l}$ witch $L^{2}$. The length of the ligament $L$ must be less than $2 r_{p}$, where $r_{p}$ : plastic zone radius.

$L<2 r_{p}$

In order to minimize edge effects some authors suggest that the width of the specimen $w$, must three times greater than the ligament size. These restrictions concern the size of the ligament $L$, which conditions the dimensions of testing specimen, they are expressed as:

$3-5 B<\min \left(2 r_{p}, w / 3\right)$.

The identification of the limit load $P_{L}$ to maximum load allows to find the result from Hill's analysis on DENT specimen. This hypothesis states that rupture occurs after the limit load. The maximum load and the initial ligament are related linearly as:

$P_{L}=p \sigma_{y} L B$

$p$ : confinement ratio; $\sigma_{y}$ : threshold plasticity of noncracked specimen.

The maximum average load in the ligament $\left(\sigma_{m m}\right)$ is defined as the ratio between maximum load and the initial ligament surface:

$\sigma_{m m}=\frac{P_{L}}{L B}=p \sigma_{y}$.

When Eq. (5) is verified, the plane stress state is then guaranteed. When $\sigma_{m m}>p \sigma_{y}$, a stress triaxiality is observed. The confinement ratio $p$ is equal to $2 / \sqrt{3}$ for DENT specimen and $p=1$ for SENT specimen. Thus, when the plane stress conditions prevail in the ligament of SENT specimens, the maximum average load is equivalent to the plasticity stress threshold $\sigma_{y}$.

$\sigma_{m m}=\frac{F_{\max }}{L B}=\sigma_{y}$

The evolution of this stress obtained from our numerical results with respect to the ligament length is show in the results simulation. 


\section{Numerical analysis}

The simulation of experimental testing of rupture have been made by finite element code "ABAQUS".

The analysis is made under plane stress conditions. The material is thermoplastic plasticized acrylic silicon polymer. Its temperature of transition is $85{ }^{\circ} \mathrm{C}$, due to the complex behavior of this polymer, a numerical approach has been adopted introducing elastoplastic behavior. Porous ratio $v=0.499$ assuring the material is incompressible, and, in order to really simulate the behavior of the material the data base stress-strain is introduced as material property (Fig. 3).

\subsection{Methodology}

A batch of cracked SENT specimen has been modelised.

Table 1 summonses the specimen dimension and the speed of solicitations witch a thickness of $0.4 \mathrm{~mm}$ and for three different lengths) $18 \mathrm{~mm}, 24 \mathrm{~mm}$ and $30 \mathrm{~mm}$ (witch constant length equals to $100 \mathrm{~mm}$.

Fig. 4 shows a sample of stress-strain curves obtained with $w=24 \mathrm{~mm}$.

\subsection{Meshing and boundary conditions}

Due to the symmetry only one-half of the SENT specimens has been modelized. The impose null displacement at nodes located on the ligament allows to introduce initial crack length.

The load is simulated by imposing a displacement of nodes located at the edge of specimen.

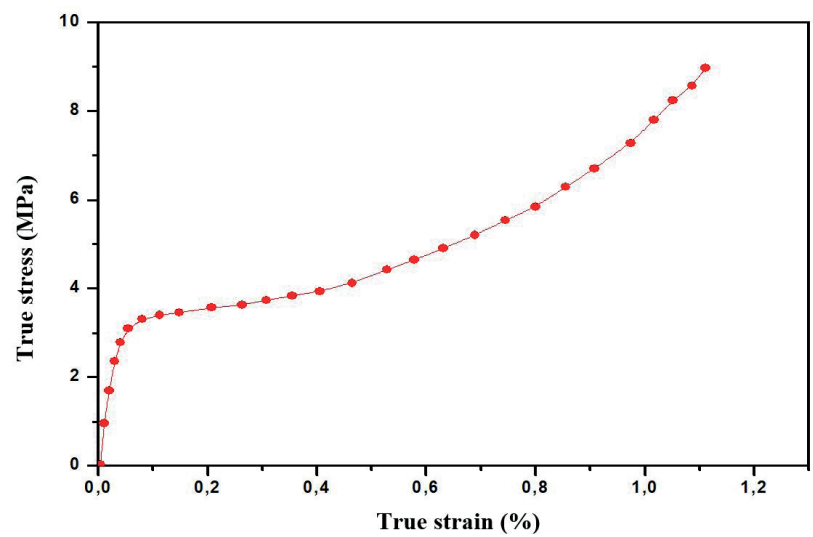

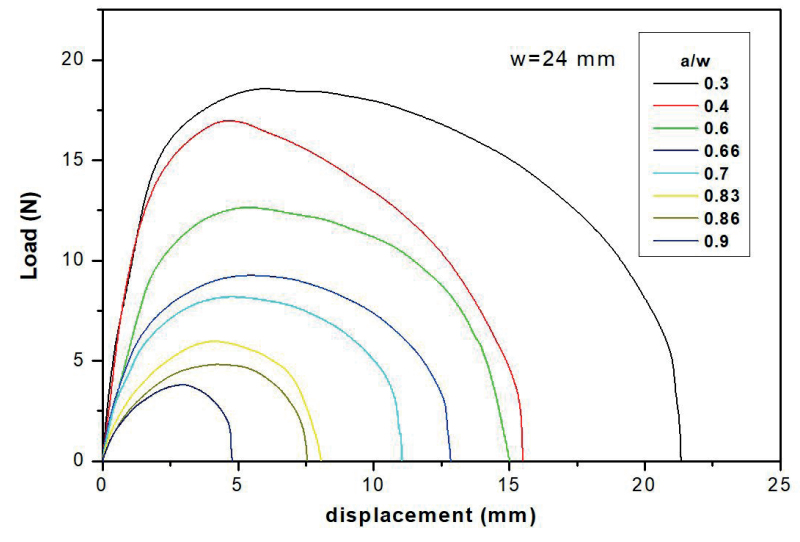

Fig. 4 Stress-strain curve of $w=24 \mathrm{~mm}$

This displacement is incremental with $0.2 \mathrm{~mm}$ step increment. This displacement is imposed on the half of the geometry of the specimen which corresponds to a double displacement for the total geometry of the specimen. The critical displacement value of initiation is then over ridded.

In this study, the elements used are quadrilaterals with 8 nodes and they are 198 in number.

In a first step, we took up this mesh by using quadrilateral elements with 8 nodes which make it possible to consider the state of plane stresses (CPS8). The calculation takes about 5 minutes.

In order to optimize the modeling time, the elements used were quadrilateral node type. The number of integration points of each element is equal to 4 (CPS4). Each node has two degree of freedom which are plane displacements $u_{x}$ and $u_{y}$ (Fig. 5).

\section{Results and discussion}

From the stress-strain curves detained from SENT specimen tests $a, b$, c (Fig. 4). The limit value $L^{*}$ has to be determined. First, the evolution of maximum load witch respect to the initial ligament length is studied (Fig. 6).

In this case, the existing relation is effectively linear. This accordance with the results found in literature [9] and [10]. The regression carried out on all the values of maximum load allow to obtain the following relation.

$$
F_{\text {max }}=1.171 L
$$

Fig. 3 Real stress-strain curve for speed of $50 \mathrm{~mm} / \mathrm{min}$

Table 1 SENT specimen dimension

\begin{tabular}{lccc}
\hline speed $(\mathrm{mm} / \mathrm{min})$ & width $(\mathrm{mm})$ & $a / w$ & Designation \\
\hline 50 & 18 & $0.5-0.55-0.66-0.72-0.77-0.83-0.88-0.94$ & SENT. $a$ \\
50 & 24 & $0.3-0.4-0.6-0.66-0.7-0.76-0.83-0.86-0.9$ & SENT. $b$ \\
50 & 30 & $0.6-0.66-0.76-0.83-0.86-.93$ & SENT. $c$ \\
\hline
\end{tabular}




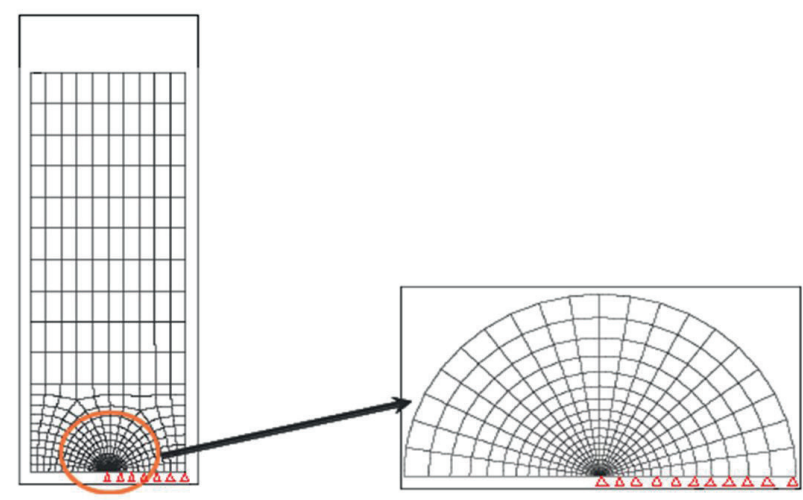

Fig. 5 Meshing and boundary conditions.

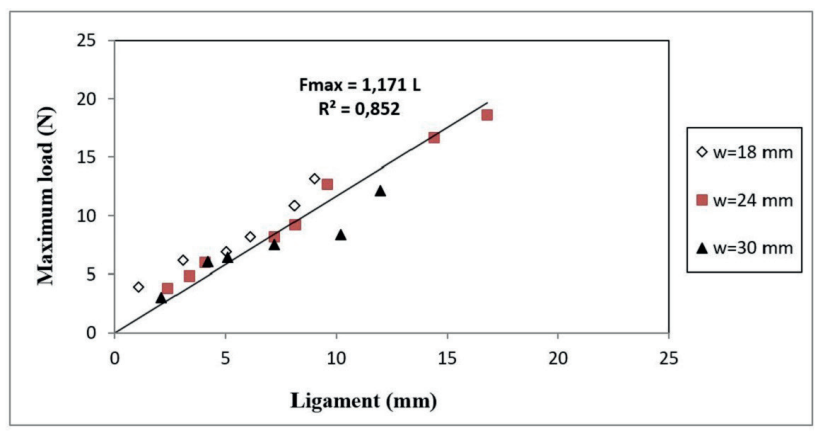

Fig. 6 Maximum load as function of initial ligament size

The identification of Eq. (9) with Eq. (6) allow to obtain the confinement ratio $p$, reminding that the threshold stress is $3 \mathrm{MPa}$ and the average value of the thickness is $0.42 \mathrm{~mm}$. The calculate value of $\mathrm{p}$ is 1.42 thus $p$ is greater than $1(p>1)$ which corresponds to rigid material perfectly plastic. This ratio can be greater than 1.42 when the maximum load is reached. This value can be explained by the stress factor is overestimated and then suggests that the limit load should be less than $F_{\text {max }}$.

The average maximum stress $\sigma_{m m}$ varies in two steps with respect to the ligament as shown in Fig. 7.

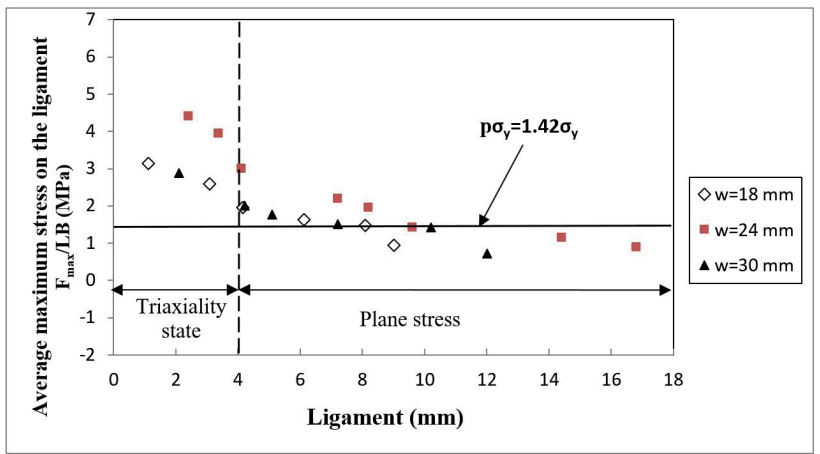

Fig. 7 Evolution of average maximum stress on the ligament with respect to initial ligament length
Then is a zone where its value is constant equivalent to 1.42 times the threshold plastic stress obtained from uniaxial tension $\sigma_{y}$.

The second zone its value is greater than $1.42 \sigma_{y}$ and increases when the size of ligament decreases. This means that the ligament is not greater in size compared to the thickness of the specimen in order to maintain a pure plane stress condition. This zone is a transition region with triaxiality state.

The limit between these two zones is for about $5 \mathrm{~mm}$ ligament size. The lengths of the ligament, which can be used to determine the essential work of fracture, must be greater than this limiting value. It's noticed that this value is four time greater than the thickness (4 thickness) of the specimen which equivalent to $1.68 \mathrm{~mm}$. The lower limit does not follow the criteria $L^{*}=3-5 \mathrm{~B}$. This observation is also that made by [11-14]. The conclusion obtained confirms the idea that this lower limit $L^{*}$ is not universal. It seems to be dependent of the material nature.

\subsection{Determination of the essential work of fracture}

Fig. 8 shows the variation of the total specific work of fracture with respect to the initial size of the ligament in plane stress conditions for three different widths of the specimen.

We note a linear evolution of $w_{f}$ with $L$ in this domain. The regression linearity is correct in all the region of plane stress conditions for which the ligaments are greater than $4 \mathrm{~mm}$. The essential work of fracture $w_{e}$ is proportional to $L$; and, the non-essential work $w_{p l}$ is proportional to $L^{2}$ on all this region. These proportionalities exist even if $L$ is greater than $w / 3$. The values of the essential work of fracture and that of non-essential work are respectively $17.85 \mathrm{KJ} / \mathrm{m}^{2}$ and $2.344 \mathrm{KJ} / \mathrm{m}^{2}$.

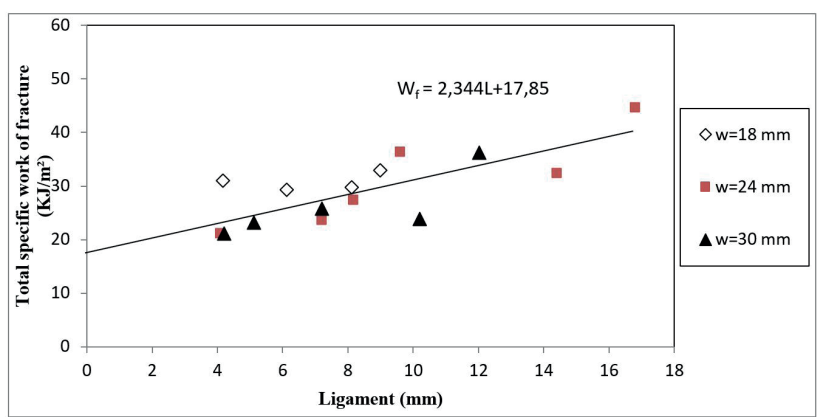

Fig. 8 Variation of total work fracture with respect to initial size of ligament for $W=18,24,30 \mathrm{~mm}$ 


\subsection{Analytical expression of $\boldsymbol{w}_{e}$ and $\boldsymbol{\beta} \boldsymbol{w}_{p l}$}

We are going to show that it is possible to evaluate the specific essential work of fracture we and also the plastic strain work around the crack tip. $\beta w_{p l}$ using equations that the terms are independent of the length of the ligament and of geometry in general: these equations are intrinsic equations.

In general, the total to rupture, $W_{f}(L)$ corresponding to the area under the stress-strain curve can be expressed as:

$W_{f}(L)=\gamma F_{\max }(L) u_{f}(L)$.

With $F_{\text {max }}(L)$, the minimum load, $u_{f}(L)$, The rupture displacement and $\gamma(L)$, a form factor.

The numerical results obtained are in good concordance with Eq. (10) as show in Fig. 9 where the factor of form is equal to 0.76 .

By the assumption, that $F_{\text {max }}$ and $u_{f}$ are dependent only on the length of the ligament and considering constant $\gamma$.

An infinitesimal variation of total work to rupture can be expressed as:

$$
d W_{f}=\gamma\left[\frac{\partial F_{\max }}{\partial L} d L u_{f}+\frac{\partial u_{f}}{\partial L} d L F_{\max }\right]
$$

According to Mai and Cotterell [7], the displacement to rupture is linked to the ligament by:

$u_{f}=\delta_{c}+k L$.

Replacing $F_{\text {max }}$ and $u_{f}$, According to Eq. (8) and Eq. (12), we obtain:

$\frac{d W_{f}}{d L}=\gamma p \sigma_{y} B\left[\delta_{c}+2 k L\right]$.

In another hand Eq. (1) and Eq. (2) gives:

$\frac{d W_{f}}{d L}=B\left[w_{e}+2 \beta w_{p l} L\right]$

If we proceed to terms identification of Eq. (13) and Eq. (14) we can obtain:

$w_{e}=\gamma p \sigma_{y} \delta_{c}$

and

$\beta w_{p l}=\gamma p \sigma_{y} \delta_{c}$

\section{References}

[1] Cotterell, B., Reddel, J. K. "The essential work of plane stress ductile fracture", International Journal of Fracture, 13(3), pp. 267-277, 1977. https://doi.org/10.1007/BF00040143

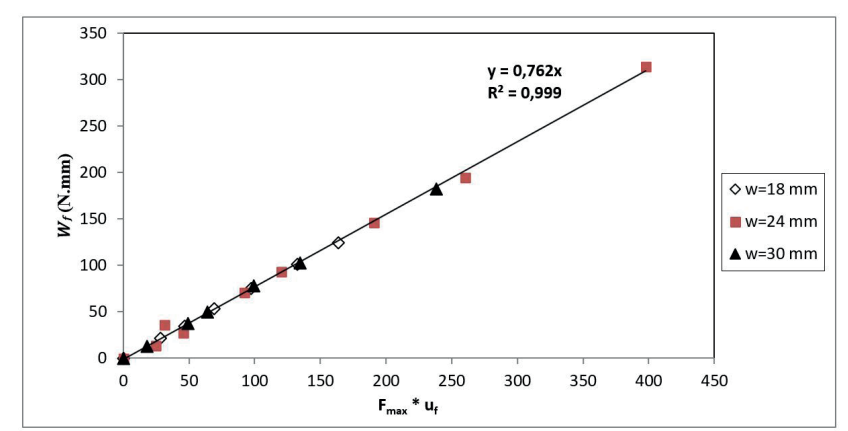

Fig. 9 Determination of the form factor

In another way, Cotterell and Reddel [1] had proposed Eq. (15). By replacing the different parameters by their numerical values of $\gamma, p, \sigma_{y}, k$ and $\delta_{c}$ we obtain $w_{e}=17.28 \mathrm{~kJ} / \mathrm{m}^{2}$ and $\beta w_{p l}=3.30 \mathrm{~kJ} / \mathrm{m}^{2}$.

The estimation of $w_{e}$ by Eq. (15) gives an average value close to the value obtained by linear regression. The two values of $\beta w_{p l}$ are in accordance.

\section{Conclusion}

The use of the essential work of fracture has been proposed. This approach is a method with multiple specimens but the conventional value obtained necessitates rupture localization. It has been observed that the specific works of rupture have the desired theoretical form in function with initial value of ligament $L^{*}$ must be greater than $4 \mathrm{~mm}$. this value is well greater than they are generally estimated which 3 to 5 times the specimen thickness. It has been observed that the applied restriction by the upper bound of dimensioning criteria is not applied here.

The values of the specific essential work of fracture are independent of the ligament length. It is the same for the non-essential work. We deduce that the plastic deformation is localized on the ligament.

Two intrinsic equations have been made in evidence: the evolution of the terms $w_{e}$ and $\beta w_{p l}$ which permit to be obtained are in good accordance with values obtained by linear regression.

[2] Broberg, K. B. "Critical Review of Some Theories in Fracture Mechanics", International Journal of Fracture Mechanics, 4(1), pp. 11-18, 1968. https://doi.org/10.1007/BF00189139 
[3] Mai, Y. W., Cottrell, B. "On the essential work of ductile fracture in polymers", International Journal Of Fracture, 32(2), pp. 105-125, 1986.

https://doi.org/10.1007/BF00019787

[4] Paton, C. A., Hashemi, S. "Plane-stress essential work of ductile fracture for polycarbonate", Journal of Materials Science, 27(9), pp. 2279-2290, 1992. https://doi.org/10.1007/BF01105033

[5] Mai, Y. W., Powell, P. "Essential work of fracture and j-integral measurements for ductile polymers", Journal of Polymer Science: Part B: Polymer Physic, 29(7), pp. 785-793, 1991. https://doi.org/10.1002/polb.1991.090290702

[6] Atkins, A. G., Mai, Y. W. "Elastic and plastic fracture: metals, polymers, ceramics, composites, biological materials", Ellis Horwood, Chichester, UK, 1985.

[7] Mai, Y. W., Cotterell, B. "Effects of pre-strain on plane stress ductile fracture in $\alpha$-brass", Journal of Materials Science, 15(9), pp. 2296-2306, 1980. https://doi.org/10.1007/BF00552319

[8] Levita, G., Parisi, L., Mcloughlin, S. "Essential work of fracture in polymer films", Journal of Materials Science, 31(6), pp. 1545-1553, 1996. https://doi.org/10.1007/BF00357863

[9] Hashemi, S. "Plane-stress fracture of polycarbonate films", Journal of Materials Science, 28(22), pp. 6178-6184, 1993.

https://doi.org/10.1007/BF00365040
[10] Chan, W. Y. F., Wililiams, J. G. "Determination of the fracture toughness of polymeric films by the essential work method", Polymer, 35(8), pp. 1666-1672, 1994. https://doi.org/10.1016/0032-3861(94)90840-0

[11] Hashemi, S. "Fracture toughness evaluation of ductile polymeric films", Journal of Materials Science, 32(6), pp. 1563-1573, 1997. https://doi.org/10.1023/A:1018582707419

[12] Maspoch, M. L., Ferrer, D., Gordillo, A., Santana, O. O., Martinez, A. B. "Effect of the specimen dimensions and the test speed on the fracture toughness of IPP by the essential work of fracture (EWF) method", Journal of Applied Polymer Science, 73(2), pp. 177-187, 1999.

https://doi.org/10.1002/(SICI)1097-4628(19990711)73:2<177::AIDAPP5>3.0.CO;2- $\% 23$

[13] Wu, J., Mai, Y. W. "The essential fracture work concept for toughness measurement of ductile polymers", Polymer Engineering and Science, 36(18), pp. 2275-2288, 1996. https://doi.org/10.1002/pen.10626

[14] Karger-Korcsis, J., Czigány, T., Moskala, E. J. "Thickness dependence of work of fracture parameters of an amorphous copolyester", Polymer, 38(18), pp. 4587-4593, 1997. https://doi.org/10.1016/S0032-3861(96)01061-0 